\title{
A study case for the analysis of asymptotic expansions beyond the tQSSA for inhibitory mechanisms in enzyme kinetics.
}

\author{
A. M. Bersani ${ }^{1}$, A. Borri ${ }^{2}$, A. Milanesi ${ }^{3}$, G. Tomassetti ${ }^{3}$, P. Vellucci $^{4}$ \\ ${ }^{1}$ Department of Mechanical and Aerospace Engineering, Sapienza University, Via Eudossiana 18, 00184 Roma \\ ${ }^{2}$ Institute for System Analysis and Computer Science "Antonio Ruberti" (IASI-CNR), Piazzale A. Moro, 700185 \\ Roma - Italy \\ ${ }^{3}$ Department of Basic and Applied Sciences for Engineering, Sapienza University, Via Antonio Scarpa 16, 00161 \\ Roma \\ ${ }^{4}$ Department of Economics, Roma Tre University, via Silvio D’Amico 77, 00145 Rome, Italy \\ ${ }^{*}$ Email address for correspondence: pierluigi.vellucci@uniroma3.it
}

Communicated by Elena De Angelis

Received on 10 28, 2018. Accepted on 07 25, 2019.

\begin{abstract}
In this paper we study the model of the chemical reaction of fully competitive inhibition and determine the appropriate parameter $\epsilon$ (related to the chemical constants of the model), for the application of singular perturbation techniques. We determine the inner and the outer solutions up to the first perturbation order and the uniform expansions. Some numerical results are discussed.
\end{abstract}

Keywords: Enzyme Kinetics, Inhibition, Singular Perturbation Theory, Asymptotic Expansions

AMS subject classification: 34D15, 34D20, 34E10, 92C45

\section{Introduction}

Mathematical modelling of chemical reactions is one of the bases of theoretical studies of actual biochemical research and has given important contributions to advances in biomedical and pharmaceutical researches.

At present, a widely used approach to model intracellular mechanisms is to break up the mechanism network into simpler subnetworks (usually called modules) and to give kinetic models for these modules in terms of systems of first order nonlinear ordinary differential equations (ODEs). A module is identified as a (discrete) protein-protein interaction. A network is a set of modules linked by inflow and outflow processes known as pathways.

The long-term goal of these studies is the integration of different modules, in order to reproduce the global behavior of a specific cell type and put the description of the dynamics and control characteristics of living organisms on physico-chemical bases.

The ODE systems governing the models are related to the rate of change of the concentrations of molecular species and depend on a set of parameters, i.e., the initial concentration of dynamical molecular species and the kinetic constants which quantitatively describe the velocity of every single reaction.

The fully competitive inhibition is one of the most common reactions characterizing any intracellular reaction network.

Inhibition is used in the production of drugs and toxic agents. Here we just give some hints about the use of inhibition in pharmacology. For a more complete analysis see [1].

Many enzymes can be inhibited by the binding of specific molecules, hence inhibiting enzyme activity is a useful control mechanism in biological systems.

Two well-known examples of inhibitors employed as drugs are penicillin, that acts by modifying the enzyme transpeptidase, thus preventing the synthesis of bacterial cell walls and consequently killing the 
bacteria, and aspirin, which modifies the enzyme cyclooxygenase, reducing the synthesis of inflammatory signals.

Let us now focus our attention on competitive inhibition, where two substrates $S_{1}$ and $S_{2}$ compete for the same enzyme $E$ : the enzyme can bind either the substrate or the inhibitor, but not both simultaneously. The competitive inhibitor is similar to the substrate, binds to the active site of the enzyme and does not allow the substrate to bind to the same active site. So a competitive inhibitor diminishes the rate of catalysis by diminishing the amount of enzyme molecules bound to a substrate. This mechanism can naturally be utilized for the production of drugs. An example is given by methotrexate, that is a chemotherapy drug which prevents the cancer cells from growing and dividing.

Actually there exists also noncompetitive inhibition, in which substrate and inhibitor can bind simultaneously two enzymes at different sites.

In the classification of inhibitors into group-specific reagents, substrate analogs and suicide inhibitors, the last ones play a fundamental role in pharmacology as they are able to inactivate the enzyme. "Monoamine oxidase deaminates neurotransmitters such as dopamine and serotonin, lowering their levels in the brain. Parkinson disease is associated with low levels of dopamine, and depression is associated with low levels of serotonin. The drug (-)deprenyl, which is used to treat Parkinson disease and depression, is a suicide inhibitor of monoamine oxidase" ( [1]).

The ODEs governing enzyme reactions are based on the so-called Michaelis-Menten kinetics (briefly described here below) and on the mass conservation principle, according to which a single molecule can interact with other species and change the activity, but the total mass must be conserved.

Let us consider a reaction where a substrate $\mathrm{S}$ binds reversibly to an enzyme $\mathrm{E}$ to form a complex $\mathrm{C}$. The complex can decay irreversibly to a product $\mathrm{P}$ and the enzyme, which is then free to bind another substrate molecule. This is summarized in the scheme

$$
E+S \underset{d}{\stackrel{a}{\rightleftharpoons}} C \stackrel{k}{\rightarrow} E+P
$$

where $a, d, k$ are kinetic parameters (supposed constant) associated with the reaction rates.

Assuming that the complex concentration is approximately constant $(\dot{C} \cong 0)$ after a short transient phase leads to the usual standard quasi-steady-state approximation (sQSSA)) [2], which is valid when the enzyme concentration is much lower than either the substrate concentration or the Michaelis constant $K_{M}=\frac{d+k}{a}$. The total QSSA (tQSSA) [3-5] is another approximation, and is valid for a broader range of parameter values covering both high and low enzyme concentrations.

The sQSSA and tQSSA are related to the asymptotic expansions of the solutions of the ODEs governing the process with respect to an appropriate parameter [2,6-8]; the topic can also be read in terms of center manifold, normal forms and bifurcation theory [9-11]. The two approximations are both developed setting the derivative of the intermediate complexes equal to zero. The theoretical justification for this assumption is given by Tikhonov's Theorems [12-17]

In Tikhonov's framework, the assumption $\dot{C}=0$ is equivalent to impose the aforementioned "appropriate parameter" (the perturbation parameter, usually denoted by $\epsilon$ ) equal to zero (see, for example, $[18,19]$.

In particular, Kumar and Josić [19] derive the mathematical expression of the center manifold for the tQSSA system just putting $\epsilon=0$. This result remains as true for a wide class of systems (including the tQSSA model) and has a mathematical explanation, related to the connection between Tikhonov's theorem and center manifold theory, as shown in the framework of Geometric Singular Perturbation Theory $[20,21]$. The theoretical investigation of the validity of the tQSSA in the case of successive reactions, where more parameters appear, relies on the theorems reported in [14], which represent the generalization of Tikhonov's Theorem $[13,17]$ to the case of more parameters.

The question is: what is the most appropriate parameter to develop an asymptotic expansion of the solutions of the ODEs modelling the reactions?

In a series of papers written by Palsson et al. [22-25], the possibility to choose the required parameter has been related to the decoupling of the eigenvalues of the Jacobian matrix of the differential system. In [24] the authors focused on chains of reactions, obtaining a block form for the resulting Jacobian matrix. 
The mechanism we are studying in this paper does not belong to this class of reactions. Nevertheless Palsson's technique was adapted to the fully competitive inhibition, too [26].

In [27], Schnell and Mendoza determine a closed formula for the sQSSA of the fully competitive inhibition, in terms of the Lambert function, as already obtained by the same authors in [28] for a single reaction.

In $[29,30]$ the authors study the tQSSA of this reaction, applying it also to double phosphorylation, which can be considered a peculiar case of inhibition, where the monophosphorylated substrate competes with the inactive substrate for the same enzyme.

In [26] the authors study the chemical reaction of inhibition and determine the appropriate parameter for the application of Tikhonov's Theorem, compute explicitly the equations of the center manifold of the system and find sufficient conditions to guarantee that in the phase space the curves which relate the behavior of the complexes to the substrates by means of the tQSSA tend asymptotically to the center manifold of the system. In other words, the above quoted paper gives another example of connection among Tikhonov's Theorem, center manifold and tQSSA, after the fundamental article [20].

As already observed, any QSSA can be interpreted as the leading order term of an asymptotic expansion of the solutions with respect of an appropriate parameter.

In this paper, we determine the asymptotic expansions beyond the tQSSA, up to the 1-st order in the parameter $\epsilon$ (introduced in [26], adopting Palsson's technique) for the inner and outer solutions and the corresponding uniform expansions.

The paper is organized in the following way: in Section 2 we recall the most important mathematical background concerning Palsson's theory. In Section 3 we recall the mathematical model of the enzymatic inhibition reaction and its adimensionalization, as done in [26], arriving at a suitable perturbation parameter $\epsilon$ which will be used in Section 4, in a study case, to determine the 0-th and the 1-st order asymptotic expansions for the inner and outer solutions and the corresponding uniform expansions, obtained by means of singular perturbation techniques. Numerical results are shown, for different values of $\epsilon$. Section 5 contains some conclusions, problems and perspectives.

\section{Preliminary results and notations - Palsson's technique}

As already observed in the previous Section, the tQSSA not only is much more efficient than the sQSSA, since it is valid in a much wider set of parameters and initial conditions, but also is a much more natural approximation. This fact can be confirmed considering the studies by Palsson and collaborators (in particular see [22]), where the authors are able to determine a sufficient condition for the validity of any QSSA in terms of the trace and the determinant of the Jacobian matrix $J$ of the system of nonlinear ODEs, governing the mechanism, with $J$ evaluated in its stationary point.

In their paper [22] Palsson and Lightfoot consider a generalized Michaelis-Menten reaction:

$$
\stackrel{I}{\rightarrow} S+E \underset{d}{\stackrel{a}{\rightleftharpoons}} C \underset{b}{\stackrel{k}{\rightleftharpoons}} P+E \stackrel{R}{\rightarrow},
$$

where the complex $C$ breaks down reversibly to form the product $P$ and to regenerate the free enzyme $E$ and where the input $I$ and the removal $R$ represent exchanges of mass across the system boundaries.

Of course the irreversible Michaelis-Menten reaction is a particular case of the above process.

In [22] the authors adopt the following techniques:

1. They use suitable scaled variables and concentrate individual parameters into the smallest number of combinations. In doing this, they introduce dimensionless parameters that separate two well distinguished categories: the ones measuring the concentrations and the ones related only to kinetic parameters. As to time, they set $\tau=a E_{T} t$.

2. They find the Jacobian matrix $J$ of the differential system, evaluate $J$ in its stationary point and find $\operatorname{det}(J)$ and $\operatorname{tr}(J)$

3. Finally they find the eigenvalues $\lambda_{1}, \lambda_{2}$ which are given by 


$$
\lambda_{1,2}=\frac{1}{2}\left[\operatorname{tr}(J) \pm \sqrt{\operatorname{tr}(J)^{2}-4 \operatorname{det}(J)}\right]
$$

4. By setting $\tau_{1}=\left|\frac{1}{\lambda_{1}}\right|$ (slower timescale) and $\tau_{2}=\left|\frac{1}{\lambda_{2}}\right|$ (faster timescale), it follows that, if $\frac{4 \operatorname{det}(J)}{\operatorname{tr}^{2}(J)}$ is small, then

$$
\left|\lambda_{2}\right| \ll\left|\lambda_{1}\right| \quad \text { i.e. } \quad \tau_{1} \ll \tau_{2} .
$$

Thus the two timescales are well separated if

$$
\frac{\operatorname{det}(J)}{\operatorname{tr}^{2}(J)} \ll 1
$$

which is a sufficient condition for the existence of fast and slow variables.

5. The authors prove that upon condition (2) the time constants may be approximated by

$$
\tau_{1} \sim\left|\frac{\operatorname{tr}(J)}{\operatorname{det}(J)}\right| ; \quad \tau_{2} \sim\left|\frac{1}{\operatorname{tr}(J)}\right| .
$$

Let us now apply Palsson's technique to the classical Michaelis-Menten kinetics with total substrate. For notation convenience we will use the same variable names to denote both a chemical species and its concentration. For example, $E$ denotes both an enzyme and its concentration.

The system of equations, called full system, governing the scheme represented in (1), is formed by two independent equations:

$$
\left\{\begin{array}{l}
\frac{d S}{d t}=-a\left(E_{T}-C\right) S+d C \\
\frac{d C}{d t}=a\left(E_{T}-C\right) S-(d+k) C
\end{array}\right.
$$

with the initial conditions

$$
S(0)=S_{T}, \quad C(0)=0
$$

and the conservation laws

$$
E+C=E_{T}, \quad S+C+P=S_{T} .
$$

The initial conditions give the concentrations of $S$ and $C$ at the beginning of the reaction, and their time development is described by the ODEs, while $E$ and $P$ are linked to $S$ and $C$ through the conservation laws.

Introducing the total substrate $\bar{S}(t)=S(t)+C(t),(3)$ then become

$$
\left\{\begin{array}{l}
\frac{d \bar{S}}{d t}=-k C \\
\frac{d C}{d t}=a\left[C^{2}-\left(E_{T}+\bar{S}+K_{M}\right) C+E_{T} \bar{S}\right] \\
\bar{S}(0)=S_{T} \\
C(0)=0
\end{array}\right.
$$


with conservation laws

$$
E+C=E_{T}, \quad \bar{S}+P=S_{T} .
$$

It is easy to see that the origin $X^{*}:=\left(s^{*}, C^{*}\right)=(0,0)$ is the only stationary point of the system, with Jacobian matrix at the origin

$$
J(0,0)=\left(\begin{array}{cc}
0 & -k \\
a E_{T}-a\left(E_{T}+K_{M}\right)
\end{array}\right) .
$$

An easy computation shows that

$$
|J-\lambda \mathbb{I}|=\lambda^{2}+a\left(E_{T}+K_{M}\right) \lambda+k a E_{T},
$$

so that the eigenvalues are

$$
\begin{aligned}
\lambda_{1,2}= & \frac{-a\left(E_{T}+K_{M}\right) \pm \sqrt{a^{2}\left(E_{T}+K_{M}\right)^{2}-4 a k E_{T}}}{2}= \\
& \frac{a\left(E_{T}+K_{M}\right)}{2}\left(-1 \pm \sqrt{1-\frac{4 K E_{T}}{\left(E_{T}+K_{M}\right)^{2}}}\right)
\end{aligned}
$$

where $\frac{4 K E_{T}}{\left(E_{T}+K_{M}\right)^{2}}=\frac{4 \operatorname{det}(J)}{\operatorname{tr}^{2}(J)}$ and $K=\frac{k}{a}$ is the Van Slyke-Cullen constant. Now it is clear that

$$
\frac{K E_{T}}{\left(E_{T}+K_{M}\right)^{2}} \ll 1 \Longrightarrow\left|\lambda_{1}\right| \ll\left|\lambda_{2}\right|
$$

and we have a sufficient condition for the separation of time scales.

Moreover we could choose $\tilde{\epsilon}=\frac{K E_{T}}{\left(E_{T}+K_{M}\right)^{2}}$ as perturbation parameter.

It easy to show that $\tilde{\epsilon}<\frac{1}{4}$ for every choice of data.

However, in order to follow $[3,8,31]$, we take

$$
\epsilon=\frac{K E_{T}}{\left(E_{T}+K_{M}+S_{T}\right)^{2}}
$$

as perturbation parameter (note that $\epsilon<\tilde{\epsilon}$ ).

Thus Palsson's results give a wide range of parameters and initial conditions assuring the validity of the tQSSA and imply that the perturbation parameter $\epsilon$ given in (10) arises as the natural parameter to be used in any efficient quasi-steady state approximation.

\section{Mathematical model of the inhibition mechanism}

A fully competitive inhibition reaction, where an inhibitor $S_{2}$ competes with a substrate $S_{1}$ for the same enzyme $E$, is summarized by the following scheme

$$
\left\{\begin{array}{l}
E+S_{1} \underset{d_{1}}{\stackrel{a_{1}}{\rightleftharpoons}} C_{1} \stackrel{k_{1}}{\longrightarrow} E+P_{1} \\
E+S_{2} \underset{d_{2}}{\stackrel{a_{2}}{\rightleftharpoons}} C_{2} \stackrel{k_{2}}{\longrightarrow} E+P_{2},
\end{array}\right.
$$

where $P_{1}, P_{2}$ are the reaction products and $C_{1}, C_{2}$ the enzyme-substrate complexes. In this kind of reaction, it is clear that $S_{1}$ and $S_{2}$ have the same role of reciprocal inhibitors $\left(S_{1}\right.$ inhibits $S_{2}$ as $S_{2}$ inhibits $S_{1}$ ). Introducing the total substrates

$$
\overline{S_{1}}=S_{1}+C_{1}, \quad \overline{S_{2}}=S_{2}+C_{2},
$$


by conservation laws we have

$$
\overline{S_{1}}+P_{1}=S_{1 T}, \overline{S_{2}}+P_{2}=S_{2 T}, \quad E+C_{1}+C_{2}=E_{T} .
$$

Then we can write the following Cauchy Problem:

$$
\left\{\begin{array}{l}
\frac{d \overline{S_{1}}}{d t}=-k_{1} C_{1} \\
\frac{d C_{1}}{d t}=a_{1}\left[\left(\overline{S_{1}}-C_{1}\right)\left(E_{T}-C_{1}-C_{2}\right)-K_{1 M} C_{1}\right] \\
\frac{d \overline{S_{2}}}{d t}=-k_{2} C_{2} \\
\frac{d C_{2}}{d t}=a_{2}\left[\left(\overline{S_{2}}-C_{2}\right)\left(E_{T}-C_{1}-C_{2}\right)-K_{2 M} C_{2}\right] \\
\overline{S_{1}}(0)=S_{1 T} \quad ; \quad \overline{S_{2}}(0)=S_{2 T} \quad ; \quad C_{1}(0)=C_{2}(0)=0
\end{array}\right.
$$

where

$$
K_{1 M}=\frac{d_{1}+k_{1}}{a_{1}}, \quad K_{2 M}=\frac{d_{2}+k_{2}}{a_{2}}
$$

are the Michaelis constants.

It is easy to see that the origin $X^{*}:=\left({\overline{S_{1}}}^{*}, C_{1}^{*},{\overline{S_{2}}}^{*}, C_{2}^{*}\right)=(0,0,0,0)$ is the only fixed point of the system. The linear approximation of the system around $X^{*}$ is represented by the following system:

$$
\left\{\begin{array}{l}
\frac{d \overline{S_{1}}}{d t}=-k_{1} C_{1} \\
\frac{d C_{1}}{d t}=a_{1}\left[E_{T} \overline{S_{1}}-E_{T} C_{1}-K_{1 M} C_{1}\right] \\
\frac{d \overline{S_{2}}}{d t}=-k_{2} C_{2} \\
\frac{d C_{2}}{d t}=a_{2}\left[E_{T} \overline{S_{2}}-E_{T} C_{2}-K_{2 M} C_{2}\right]
\end{array}\right.
$$

with Jacobian at the origin

$$
J(0,0,0,0)=\left(\begin{array}{cccc}
0 & -k_{1} & 0 & 0 \\
a_{1} E_{T}-a_{1}\left(E_{T}+K_{1 M}\right) & 0 & 0 \\
0 & 0 & 0 & -k_{2} \\
0 & 0 & a_{2} E_{T}-a_{2}\left(E_{T}+K_{2 M}\right)
\end{array}\right),
$$

characterized by two blocks. The Jacobian allows the separation between the eigenvalues of the first reaction and the eigenvalues of the second reaction, so that we can apply Palsson's technique to find the perturbation parameter $\epsilon$, as one can see considering the results obtained in [26]. An easy computation shows that

$$
\begin{aligned}
|D-\lambda \mathbb{I}| & =\left[\lambda^{2}+a_{1}\left(E_{T}+K_{1 M}\right) \lambda+k_{1} a_{1} E_{T}\right]\left[\lambda^{2}+\right. \\
& \left.+a_{1}\left(E_{T}+K_{2 M}\right) \lambda+k_{2} a_{2} E_{T}\right]
\end{aligned}
$$

then the eigenvalues are

$$
\begin{gathered}
\left(\lambda_{ \pm}\right)_{i}=\frac{-a_{i}\left(E_{T}+K_{i M}\right) \pm \sqrt{a_{i}^{2}\left(E_{T}+K_{i M}\right)^{2}-4 a_{i} k_{i} E_{T}}}{2}= \\
\frac{a_{i}\left(E_{T}+K_{i M}\right)}{2}\left(-1 \pm \sqrt{1-\frac{4 K_{i} E_{T}}{\left(E_{T}+K_{i M}\right)^{2}}}\right)
\end{gathered}
$$

where we have denoted the Van Slyke-Cullen constants by $K_{i}=\frac{k_{i}}{a_{i}}, i=1,2$. Now it is clear that

$$
\frac{4 K_{i} E_{T}}{E_{T}+K_{i M}} \ll 1 \Longrightarrow\left|\left(\lambda_{+}\right)_{i}\right| \ll\left|\left(\lambda_{-}\right)_{i}\right|
$$


$i=1,2$, i.e., according to Palsson [24], we have a sufficient condition for the separation of time scales.

We could then choose

$$
\tilde{\epsilon}:=\max \left\{\tilde{\epsilon}_{i}:=\frac{K_{i} E_{T}}{\left(E_{T}+K_{i M}\right)^{2}} ; i=1,2\right\},
$$

as perturbation parameter. It easy to show that $\tilde{\epsilon}_{i}<\frac{1}{4}$ for every choice of data. However, in order to follow the paper [8], it is more convenient to take

$$
\epsilon=\max \left\{\epsilon_{i}:=\frac{K_{i} E_{T}}{\left(E_{T}+K_{i M}+S_{i T}\right)^{2}} ; i=1,2\right\}<\frac{1}{4}
$$

as perturbation parameter (note that $\epsilon_{i}<\tilde{\epsilon}_{i}$ ). Furthermore, thanks to the symmetry of the problem, it is sufficient to consider just one case (see again [26]): we can suppose that $\epsilon=\epsilon_{1}$. With this choice, the change of variables $\overline{S_{i}}=\alpha_{i} s_{i}, C_{i}=\beta_{i} c_{i}, t=\gamma \tau$, with

$$
\alpha_{i}=S_{i T}, \quad \beta_{i}=\frac{E_{T} S_{i T}}{E_{T}+K_{i M}+S_{i T}}, \gamma=\frac{1}{a_{1}\left(E_{T}+K_{1 M}+S_{1 T}\right)},
$$

$i=1,2$, provides an adimensionalization of the model equations (see [6-8,26,32]:

$$
\left\{\begin{aligned}
\frac{\alpha_{1}}{\gamma} \frac{d \overline{s_{1}}}{d \tau} & =-k_{1} \beta_{1} c_{1} \\
\frac{\beta_{1}}{\gamma} \frac{d c_{1}}{d \tau} & =a_{1}\left[\beta_{1}^{2} c_{1}^{2}-\left(E_{T}+\alpha_{1} \overline{s_{1}}-\beta_{2} c_{2}+K_{1 M}\right) \beta_{1} c_{1}+\right. \\
& \left.-\alpha_{1} \overline{s_{1}} \beta_{2} c_{2}+\alpha_{1} \overline{s_{1}} E_{T}\right] \\
\frac{\alpha_{2}}{\gamma} \frac{d \overline{s_{2}}}{d \tau} & =-k_{2} \beta_{2} c_{2} \\
\frac{\beta_{2}}{\gamma} \frac{d c_{2}}{d \tau} & =a_{2}\left[\beta_{2}^{2} c_{2}^{2}-\left(E_{T}+\alpha_{2} \overline{s_{2}}-\beta_{1} c_{1}+K_{2 M}\right) \beta_{2} c_{2}+\right. \\
& \left.-\alpha_{2} \overline{s_{2}} \beta_{1} c_{1}+\alpha_{2} \overline{s_{2}} E_{T}\right]
\end{aligned}\right.
$$

thanks to which we obtain the system of equations for the inner solutions:

$$
\left\{\begin{aligned}
\frac{d \overline{s_{1}}}{d \tau} & =-\epsilon c_{1} \\
\frac{d c_{1}}{d \tau} & =\sigma_{1} \eta_{1} c_{1}^{2}-\left(\eta_{1}+\kappa_{1 M}\right) c_{1}-\sigma_{1} \overline{s_{1}} c_{1}+\sigma_{2} \eta_{1} c_{1} c_{2}+\overline{s_{1}}-\sigma_{2} c_{2} \overline{s_{1}} \\
& =\left(s_{1}-\eta_{1} c_{1}\right)\left(1-\sigma_{1} c_{1}-\sigma_{2} c_{2}\right)-\kappa_{1 M} c_{1} \\
\frac{d \overline{s_{2}}}{d \tau} & =-\epsilon \frac{k_{2} \eta_{2}}{k_{1} \eta_{1}} c_{2} \\
\frac{d c_{2}}{d \tau} & =\frac{a_{2}}{a_{1}}\left[\eta_{1} \sigma_{2} c_{2}^{2}-\left(\eta_{1}+\frac{K_{2 M}}{K_{1 M}} \kappa_{1 M}\right) c_{2}+\right. \\
& \left.+\left(\eta_{1} \sigma_{1} c_{1}-\frac{\eta_{1}}{\eta_{2}} \sigma_{2} \overline{s_{2}}\right) c_{2}+\frac{\eta_{1}}{\eta_{2}} \overline{s_{2}}-\frac{\sigma_{1} \eta_{1}}{\eta_{2}} \overline{s_{2}} c_{1}\right] \\
& =\frac{a_{2}}{a_{1}}\left[\left(\frac{\eta_{1}}{\eta_{2}} s_{2}-\eta_{1} c_{2}\right)\left(1-\sigma_{1} c_{1}-\sigma_{2} c_{2}\right)-\frac{K_{2 M}}{K_{1 M}} \kappa_{1 M} c_{2}\right]
\end{aligned}\right.
$$

where

$$
\sigma_{i}=\frac{S_{i T}}{E_{T}+K_{i M}+S_{i T}} ; \quad \eta_{i}=\frac{E_{T}}{E_{T}+K_{i M}+S_{i T}} ; \quad \kappa_{i M}=\frac{K_{i M}}{E_{T}+K_{i M}+S_{i T}}, i=1,2
$$

(note that $\sigma_{i}+\eta_{i}+\kappa_{i M}=1$ ), while, for the system of equations that gives the outer solution, setting

$$
\bar{\gamma}:=\frac{1}{k_{1} \eta_{1}}
$$

and noting that putting $T=\frac{t}{\bar{\gamma}}$, we see that $T=\frac{\gamma}{\bar{\gamma}} \tau=\epsilon \tau$, we obtain [26]

$$
\left\{\begin{aligned}
\frac{d \overline{s_{1}}}{d T} & =-c_{1} \\
\epsilon \frac{d c_{1}}{d T} & =\sigma_{1} \eta_{1} c_{1}^{2}-\left(\eta_{1}+\kappa_{1 M}\right) c_{1}-\sigma_{1} \overline{s_{1}} c_{1}+\sigma_{2} \eta_{1} c_{1} c_{2}+\overline{s_{1}}-\sigma_{2} c_{2} \overline{s_{1}} \\
& =\left(s_{1}-\eta_{1} c_{1}\right)\left(1-\sigma_{1} c_{1}-\sigma_{2} c_{2}\right)-\kappa_{1 M} c_{1} \\
\frac{d \overline{s_{2}}}{d T} & =-\frac{k_{2} \eta_{2}}{k_{1} \eta_{1}} c_{2} \\
\epsilon \frac{d c_{2}}{d T} & =\frac{a_{2}}{a_{1}}\left[\eta_{1} \sigma_{2} c_{2}^{2}-\left(\eta_{1}+\frac{K_{2 M}}{K_{1 M}} \kappa_{1 M}\right) c_{2}+\right. \\
& \left.+\left(\eta_{1} \sigma_{1} c_{1}-\frac{\eta_{1}}{\eta_{2}} \sigma_{2} \overline{s_{2}}\right) c_{2}+\frac{\eta_{1}}{\eta_{2}} \overline{s_{2}}-\frac{\sigma_{1} \eta_{1}}{\eta_{2}} \overline{s_{2}} c_{1}\right] \\
& =\frac{a_{2}}{a_{1}}\left[\left(\frac{\eta_{1}}{\eta_{2}} s_{2}-\eta_{1} c_{2}\right)\left(1-\sigma_{1} c_{1}-\sigma_{2} c_{2}\right)-\frac{K_{2 M}}{K_{1 M}} \kappa_{1 M} c_{2}\right] .
\end{aligned}\right.
$$


Putting $\epsilon=0$, and applying Tikhonov's theory, we obtain the reduced system of equations, a system which is of differential-algebraic (DAE) type:

$$
\left\{\begin{array}{l}
\frac{d \overline{s_{1}}}{d T}=-c_{1} \\
\sigma_{1} \eta_{1} c_{1}^{2}-\left(\eta_{1}+\kappa_{1 M}\right) c_{1}-\sigma_{2} c_{2} \overline{s_{1}}-\sigma_{1} \overline{s_{1}} c_{1}+\sigma_{2} \eta_{1} c_{1} c_{2}+\overline{s_{1}}=0 \\
\frac{d \overline{s_{2}}}{d T}=-\frac{k_{2} \eta_{2}}{k_{1} \eta_{1}} c_{2} \\
\eta_{1} \sigma_{2} c_{2}^{2}-\left(\eta_{1}+\frac{K_{2 M}}{K_{1 M}} \kappa_{1 M}\right) c_{2}-\frac{\sigma_{1} \eta_{1}}{\eta_{2}} \overline{s_{2}} c_{1}+ \\
+\left(\eta_{1} \sigma_{1} c_{1}-\frac{\eta_{1}}{\eta_{2}} \sigma_{2} \overline{s_{2}}\right) c_{2}+\frac{\eta_{1}}{\eta_{2}} \overline{s_{2}}=0
\end{array}\right.
$$

which corresponds to the tQSSA of the system $[26,29,33]$.

\section{Asymptotic Expansions}

\subsection{Inner expansions}

From now on we will write $s_{i}$ instead of $\overline{s_{i}}, i=1,2$.

Starting from the system of equation for the inner solutions (23) and setting

$$
\begin{aligned}
& s_{1}=s_{10}+\epsilon s_{11}+o(\epsilon), \quad c_{1}=c_{10}+\epsilon c_{11}+o(\epsilon), \\
& s_{2}=s_{20}+\epsilon s_{21}+o(\epsilon), \quad c_{2}=c_{20}+\epsilon c_{21}+o(\epsilon),
\end{aligned}
$$

we obtain, for the 0 -th order,

$$
\begin{gathered}
\left\{\begin{array}{l}
\frac{d s_{10}}{d \tau}=\frac{d s_{20}}{d \tau}=0 \\
s_{10}(0)=s_{20}(0)=1
\end{array} \Longrightarrow s_{10}(\tau)=s_{20}(\tau)=1\right. \\
\left\{\begin{array}{l}
\frac{d c_{10}}{d \tau}=\left(1-\eta_{1} c_{10}\right)\left(1-\sigma_{1} c_{10}-\sigma_{2} c_{20}\right)-\kappa_{1 M} c_{10} ; \\
\frac{d c_{20}}{d \tau}=\frac{a_{2}}{a_{1}}\left[\left(\frac{\eta_{1}}{\eta_{2}}-\eta_{1} c_{20}\right)\left(1-\sigma_{1} c_{10}-\sigma_{2} c_{20}\right)-\frac{K_{2 M}}{K_{1 M}} \kappa_{1 M} c_{20}\right] \\
c_{10}(0)=c_{20}(0)=0
\end{array}\right.
\end{gathered}
$$

and for the 1-st order

$$
\begin{cases}\frac{d s_{11}}{d \tau} & =-c_{10} \\ \frac{d c_{11}}{d \tau} & =\left(s_{11}-\eta_{1} c_{11}\right)\left(1-\sigma_{1} c_{10}-\sigma_{2} c_{20}\right)+\left(s_{10}-\eta_{1} c_{10}\right)\left(-\sigma_{1} c_{11}-\sigma_{2} c_{21}\right)-\kappa_{1 M} c_{11} \\ \frac{d s_{21}}{d \tau} & =-\frac{k_{2} \eta_{2}}{k_{1} \eta_{1}} c_{20} \\ \frac{d c_{21}}{d \tau} & =\frac{a_{2}}{a_{1}}\left[\left(\frac{\eta_{1}}{\eta_{2}} s_{20}-\eta_{1} c_{20}\right)\left(-\sigma_{1} c_{11}-\sigma_{2} c_{21}\right)+\left(\frac{\eta_{1}}{\eta_{2}} s_{21}-\eta_{1} c_{21}\right)\left(1-\sigma_{1} c_{10}-\sigma_{2} c_{20}\right)+\right. \\ & \left.-\frac{K_{2 M}}{K_{1 M}} \kappa_{1 M} c_{21}\right] \\ c_{11}(0) & =c_{21}(0)=s_{11}(0)=s_{21}(0)=0\end{cases}
$$

A Study Case In order to determine explicit solutions of the system (29), we impose some conditions on the parameters and initial conditions: $K_{1 M}=K_{2 M}=K_{M}, a_{1}=a_{2}=a, S_{1 T}=S_{2 T}=S_{T}$, from which $a_{1}=a_{2}=a, \kappa_{1 M}=\kappa_{2 M}=\kappa_{M}, \sigma_{1}=\sigma_{2}=\sigma$ and $\eta_{1}=\eta_{2}=\eta$.

Let us observe that these conditions are a bit restrictive, leaving only three degrees of freedom: $d_{1} ; k_{1} ; k_{2}$, while $d_{2}$ is related to the other rates by the relation $d_{2}=k_{1}-k_{2}+d_{1}$. Nevertheless the study case can be useful to capture the quantitative behaviour of the system, by means of explicit approximating formulas. 
Let us first study the 0-th order expansions. We have:

$$
\left\{\begin{array}{l}
\frac{d c_{10}}{d \tau}=\left(1-\eta c_{10}\right)\left[1-\sigma\left(c_{10}+c_{20}\right)\right]-\kappa_{M} c_{10} \\
\frac{d c_{20}}{d \tau}=\left(1-\eta c_{20}\right)\left[1-\sigma\left(c_{10}+c_{20}\right)\right]-\kappa_{M} c_{20} \\
c_{10}(0)=c_{20}(0)=0
\end{array}\right.
$$

Putting

$$
c_{10}+c_{20}=u, \quad c_{10}-c_{20}=v
$$

we find

$$
\left\{\begin{array}{l}
\frac{d u}{d \tau}=\eta \sigma u^{2}-(1+\sigma) u+2 \\
\frac{d v}{d \tau}=[\sigma \eta u+\sigma-1] v \\
u(0)=v(0)=0
\end{array}\right.
$$

from which

$$
u(\tau)=\frac{2}{\eta \sigma}\left[\frac{e^{\sqrt{\Delta} \tau}-1}{u_{+} e^{\sqrt{\Delta} \tau}-u_{-}}\right]
$$

where

$$
u_{ \pm}=\frac{1+\sigma \pm \sqrt{(1+\sigma)^{2}-8 \eta \sigma}}{2 \eta \sigma}, \Delta=(1+\sigma)^{2}-8 \eta \sigma>0,
$$

and $v(\tau)=0$ (singular solution).

Let us observe that

$$
u_{+}+u_{-}=\frac{1+\sigma}{\eta \sigma} \quad ; \quad u_{+}-u_{-}=\frac{\sqrt{\Delta}}{\eta \sigma} \quad ; \quad u_{+} \cdot u_{-}=\frac{2}{\eta \sigma} \quad ; \quad \frac{u_{+}-u_{-}}{u_{+}}=\frac{\sqrt{\Delta}}{\eta \sigma u_{+}}=\frac{\sqrt{\Delta} u_{-}}{2} .
$$

Thus

$$
\lim _{\tau \rightarrow+\infty} u(\tau)=u_{-}
$$

It follows that

$$
c_{10}(\tau)=c_{20}(\tau)=\frac{1}{\eta \sigma}\left[\frac{e^{\sqrt{\Delta} \tau}-1}{u_{+} e^{\sqrt{\Delta} \tau}-u_{-}}\right]=: c_{0}(\tau)
$$

and

$$
\lim _{\tau \rightarrow+\infty} c_{10}(\tau)=\lim _{\tau \rightarrow+\infty} c_{20}(\tau)=\frac{u_{-}}{2} .
$$

At order 1, starting from (29) and (33), we have

$$
\begin{gathered}
s_{11}(\tau)=\frac{-u_{+}}{2} \tau+\frac{1}{2 \eta \sigma} \ln \left(\frac{u_{+} e^{\sqrt{\Delta} \tau}-u_{-}}{u_{+}-u_{-}}\right) \sim_{\tau \rightarrow \infty} \frac{-u_{-}}{2} \tau-\frac{1}{2 \eta \sigma} \ln \left(\frac{\sqrt{\Delta} u_{-}}{2}\right)=: s_{11}^{a s}(\tau), \\
s_{21}(\tau)=\frac{k_{2}}{k_{1}} s_{11}(\tau)=\frac{k_{2}}{k_{1}}\left[\frac{-u_{+}}{2} \tau+\frac{1}{2 \eta \sigma} \ln \left(\frac{u_{+} e^{\sqrt{\Delta} \tau}-u_{-}}{u_{+}-u_{-}}\right)\right] \\
\sim_{\tau \rightarrow \infty} \frac{k_{2}}{k_{1}}\left[\frac{-u_{-}}{2} \tau-\frac{1}{2 \eta \sigma} \ln \left(\frac{\sqrt{\Delta} u_{-}}{2}\right)\right]=: s_{21}^{a s}(\tau)
\end{gathered}
$$

and

$$
s_{11}^{a s}(0)=-\frac{1}{2 \eta \sigma} \ln \left(\frac{\sqrt{\Delta} u_{-}}{2}\right) \quad ; \quad s_{21}^{a s}(0)=-\frac{k_{2}}{k_{1}}\left[\frac{1}{2 \eta \sigma} \ln \left(\frac{\sqrt{\Delta} u_{-}}{2}\right)\right] .
$$


Let us now determine $c_{11}(\tau)$ and $c_{21}(\tau)$.

Since

$$
\left\{\begin{aligned}
\frac{d\left(c_{11}+c_{21}\right)}{d \tau} & =\left[1-\sigma\left(c_{10}+c_{20}\right)\right]\left[s_{11}+s_{21}-\eta\left(c_{11}+c_{21}\right)\right]- \\
& -\sigma\left(c_{11}+c_{21}\right)\left[s_{10}+s_{20}-\eta\left(c_{11}+c_{21}\right)\right]-\kappa_{M}\left(c_{11}+c_{21}\right) \\
\frac{d\left(c_{11}-c_{21}\right)}{d \tau} & =\left[1-\sigma\left(c_{10}+c_{20}\right)\right]\left[s_{11}-s_{21}-\eta\left(c_{11}-c_{21}\right)\right]- \\
& -\sigma\left(c_{11}+c_{21}\right)\left[s_{10}-s_{20}-\eta\left(c_{11}-c_{21}\right)\right]-\kappa_{M}\left(c_{11}-c_{21}\right)
\end{aligned}\right.
$$

setting $p:=c_{11}+c_{21}$ and $q:=c_{11}-c_{21}$, we rewrite the system in the form

$$
\left\{\begin{array}{l}
\frac{d p}{d \tau}=\left(-\sigma-1+4 \eta \sigma c_{10}\right) p+\left(1-2 \sigma c_{10}\right) y s_{11} \\
\frac{d q}{d \tau}=\left(\sigma-1+2 \eta \sigma c_{10}\right) q+\left(1-2 \sigma c_{10}\right) w s_{11} \\
p(0)=q(0)=0
\end{array}\right.
$$

where $y=\left(1+\frac{k_{2}}{k_{1}}\right) ; w=\left(1-\frac{k_{2}}{k_{1}}\right)$. Solving, we obtain

$$
\left\{\begin{array}{l}
p(\tau)=e^{\int_{0}^{\tau}\left(-\sigma-1+4 \eta \sigma c_{0}(t)\right) d t}\left[y \int_{0}^{\tau}\left(1-2 \sigma c_{0}(t)\right) s_{11}(t) e^{\int_{0}^{t}\left(\sigma+1-4 \eta \sigma c_{0}(s)\right) d s} d t\right] \\
q(\tau)=e^{\int_{0}^{\tau}\left(\sigma-1+2 \eta \sigma c_{0}(t)\right) d t}\left[w \int_{0}^{\tau}\left(1-2 \sigma c_{0}(t)\right) s_{11}(t) e^{\int_{0}^{t}\left(1-\sigma-2 \eta \sigma c_{0}(s)\right) d s} d t\right]
\end{array} .\right.
$$

Since

we find

$$
\int_{0}^{\tau} c_{0}(t) d t=\frac{1}{\eta \sigma}\left[\frac{1}{u_{-}} \tau-\frac{1}{2} \ln \left(\frac{u_{+} e^{\sqrt{\Delta} \tau}-u_{-}}{u_{+}-u_{-}}\right)\right]
$$

$$
\begin{gathered}
p(\tau)=\frac{y e^{-\sqrt{\Delta} \tau}}{\left(u_{+}-u_{-} e^{-\sqrt{\Delta} \tau}\right)^{2}} \int_{0}^{\tau}\left[1-\frac{2}{\eta}\left(\frac{e^{\sqrt{\Delta} t}-1}{u_{+} e^{\sqrt{\Delta} t}-u_{-}}\right)\right] . \\
\cdot\left[-\frac{u_{+}}{2} t+\frac{1}{2 \eta \sigma} \ln \left(\frac{u_{+} e^{\sqrt{\Delta} t}-u_{-}}{u_{+}-u_{-}}\right)\right]\left(u_{+} e^{\sqrt{\Delta} t}-u_{-}\right)^{2} e^{\left(\sigma+1-\frac{4}{u_{-}}\right) t} d t .
\end{gathered}
$$

The integration steps are simple, but tedious (see, for example, [8]). Since some integrals cannot be explicitly computed, it is easier to solve numerically (38). On the other hand, in order to build the uniform expansions, according to [2,6-8,32], we need also the asymptotic behaviour of the inner solutions.

Taking into account that $c_{0}(\tau) \sim_{\tau \rightarrow+\infty} u_{-} / 2$, it is possible to show with simple computations that both the exponents in the integrating factors in (39) are negative, thus we can eventually ignore all the converging integrals in the square brackets.

Thus,

$$
p(\tau) \sim_{\tau \rightarrow+\infty} p^{a s}(\tau):=\left(1+\frac{k_{2}}{k_{1}}\right)\left(1-\sigma u_{-}\right)\left[-\frac{u_{-}}{2 \sqrt{\Delta}} \tau+\frac{1}{2 \eta \sigma \sqrt{\Delta}} \ln \left(\frac{u_{+}}{u_{+}-u_{-}}\right)+\frac{u_{-}}{2 \Delta}\right]
$$

and

$$
p^{a s}(0)=-\left(1+\frac{k_{2}}{k_{1}}\right) \frac{\left(1-\sigma u_{-}\right)}{2 \sqrt{\Delta}}\left[\frac{1}{\eta \sigma} \ln \left(\frac{u_{-} \sqrt{\Delta}}{2}\right)-\frac{u_{-}}{\sqrt{\Delta}}\right] .
$$

Analogously we see that

$$
\begin{aligned}
& q(\tau) \sim_{\tau \rightarrow+\infty} q^{a s}(\tau):=\left(1-\frac{k_{2}}{k_{1}}\right)\left(1-\sigma u_{-}\right) . \\
& \cdot\left\{\left[\frac{-u_{-}}{2} \tau+\frac{1}{2 \eta \sigma} \ln \left(\frac{u_{+}}{u_{+}-u_{-}}\right)\right] \frac{1}{1-\sigma-\eta \sigma u_{-}}+\frac{u_{-}}{2\left(1-\sigma-\eta \sigma u_{-}\right)^{2}}\right\}
\end{aligned}
$$


and

$$
q^{a s}(0)=\left(1-\frac{k_{2}}{k_{1}}\right)\left(1-\sigma u_{-}\right)\left[\frac{-1}{2 \eta \sigma\left(1-\sigma-\eta \sigma u_{-}\right)} \ln \left(\frac{\sqrt{\Delta} u_{-}}{2}\right)+\frac{u_{-}}{2\left(-1+\sigma+\eta \sigma u_{-}\right)^{2}}\right] .
$$

Finally, we obtain

$$
\begin{array}{ll}
c_{11}^{a s}(\tau)=\frac{p^{a s}(\tau)+q^{a s}(\tau)}{2} \quad ; \quad c_{21}^{a s}(\tau)=\frac{p^{a s}(\tau)-q^{a s}(\tau)}{2} \\
c_{11}^{a s}(0)=\frac{p^{a s}(0)+q^{a s}(0)}{2} \quad ; \quad c_{21}^{a s}(0)=\frac{p^{a s}(0)-q^{a s}(0)}{2}
\end{array}
$$

\subsection{Outer expansions}

Starting from the system of equations for the outer solutions (25) and setting

$$
\begin{aligned}
& c_{1}=\Gamma_{10}+\epsilon \Gamma_{11}+o(\epsilon), \quad s_{1}=\Sigma_{10}+\epsilon \Sigma_{11}+o(\epsilon) ; \\
& c_{2}=\Gamma_{20}+\epsilon \Gamma_{21}+o(\epsilon), \quad s_{2}=\Sigma_{20}+\epsilon \Sigma_{21}+o(\epsilon)
\end{aligned}
$$

we obtain the following relations:

0-th order:

$$
\left\{\begin{array}{l}
\frac{d \Sigma_{10}}{d T}=-\Gamma_{10} \\
\left(\Sigma_{10}-\eta_{1} \Gamma_{10}\right)\left(1-\sigma_{1} \Gamma_{10}-\sigma_{2} \Gamma_{20}\right)-\kappa_{1 M} \Gamma_{10}=0 \\
\frac{d \Sigma_{20}}{d T}=-\frac{k_{2} \eta_{2}}{k_{1} \eta_{1}} \Gamma_{20} \\
\left(\frac{\eta_{1}}{\eta_{2}} \Sigma_{20}-\eta_{1} \Gamma_{20}\right)\left(1-\sigma_{1} \Gamma_{10}-\sigma_{2} \Gamma_{20}\right)-\frac{K_{2 M}}{K_{1 M}} \kappa_{1 M} \Gamma_{20}=0
\end{array}\right.
$$

from which

$$
\begin{gathered}
\Gamma_{10}=\frac{\Sigma_{10}\left[\left(\eta_{1} \sigma_{2} \Sigma_{10}^{\frac{k_{2}}{k_{1}}}+\eta_{2} \sigma_{1} \Sigma_{10}\right)+\eta_{2}\left(\eta_{1}+\kappa_{1 M}\right)\right]}{2 \eta_{1}\left(\eta_{1} \sigma_{2} \Sigma_{10}^{\frac{k_{2}}{k_{1}}}+\eta_{2} \sigma_{1} \Sigma_{10}\right)}+ \\
-\frac{\Sigma_{10} \sqrt{\left[\left(\eta_{1} \sigma_{2} \Sigma_{10}^{\frac{k_{2}}{k_{1}}}+\eta_{2} \sigma_{1} \Sigma_{10}\right)+\eta_{2}\left(\eta_{1}+\kappa_{1 M}\right)\right]^{2}-4 \eta_{1} \eta_{2}\left(\eta_{1} \sigma_{2} \Sigma_{10}^{\frac{k_{2}}{k_{1}}}+\eta_{2} \sigma_{1} \Sigma_{10}\right)}}{2 \eta_{1}\left(\eta_{1} \sigma_{2} \Sigma_{10}^{\frac{k_{2}}{k_{1}}}+\eta_{2} \sigma_{1} \Sigma_{10}\right)} \\
\Gamma_{20}=\frac{\Sigma_{10}^{\frac{k_{2}}{k_{1}}}\left[\left(\eta_{1} \sigma_{2} \Sigma_{10}^{\frac{k_{2}}{k_{1}}}+\eta_{2} \sigma_{1} \Sigma_{10}\right)+\eta_{2}\left(\eta_{1}+\kappa_{1 M}\right)\right]}{2 \eta_{2}\left(\eta_{1} \sigma_{2} \Sigma_{10}^{\frac{k_{2}}{k_{1}}}+\eta_{2} \sigma_{1} \Sigma_{10}\right)}+ \\
-\frac{\sum_{10}^{\frac{k_{2}}{k_{1}}} \sqrt{\left[\left(\eta_{1} \sigma_{2} \Sigma_{10}^{k_{2}}+\eta_{2} \sigma_{1} \Sigma_{10}\right)+\eta_{2}\left(\eta_{1}+\kappa_{1 M}\right)\right]^{2}-4 \eta_{1} \eta_{2}\left(\eta_{1} \sigma_{2} \Sigma_{10}^{\frac{k_{2}}{k_{1}}}+\eta_{2} \sigma_{1} \Sigma_{10}\right)}}{2 \eta_{2}\left(\eta_{1} \sigma_{2} \Sigma_{10}^{\frac{k_{2}}{k_{1}}}+\eta_{2} \sigma_{1} \Sigma_{10}\right)}
\end{gathered}
$$


1-st order:

$$
\left\{\begin{array}{l}
\frac{d \Sigma_{11}}{d T}=-\Gamma_{11} \\
\frac{d \Gamma_{10}}{d T}=\left(\Sigma_{10}-\eta_{1} \Gamma_{10}\right)\left(-\sigma_{1} \Gamma_{11}-\sigma_{2} \Gamma_{21}\right)+ \\
+\left(\Sigma_{11}-\eta_{1} \Gamma_{11}\right)\left(1-\sigma_{1} \Gamma_{10}-\sigma_{2} \Gamma_{20}\right)-\kappa_{1 M} \Gamma_{11} \\
\frac{d \Sigma_{21}}{d T}=-\frac{k_{2} \eta_{2}}{k_{1} \eta_{1} \Gamma_{21}} \\
\frac{d \Gamma_{20}}{d T}=\frac{a_{2}}{a_{1}}\left[\left(\frac{\eta_{1}}{\eta_{2}} \Sigma_{20}-\eta_{1} \Gamma_{20}\right)\left(-\sigma_{1} \Gamma_{11}-\sigma_{2} \Gamma_{21}\right)\right]+ \\
+\frac{a_{2}}{a_{1}}\left[\left(\frac{\eta_{1}}{\eta_{2}} \Sigma_{21}-\eta_{1} \Gamma_{21}\right)\left(1-\sigma_{1} \Gamma_{10}-\sigma_{2} \Gamma_{20}\right)-\frac{K_{2 M}}{K_{1 M}} \kappa_{1 M} \Gamma_{21}\right]
\end{array}\right.
$$

with the appropriate initial conditions, respectively, to be determined by means of appropriate matching conditions.

Study Case. Let us recall that we set

$$
K_{1 M}=K_{2 M}=K_{M} ; \quad a_{1}=a_{2}=a ; \quad \kappa_{1 M}=\kappa_{2 M}=\kappa_{M} ; \quad \sigma_{1}=\sigma_{2}=\sigma ; \quad \eta_{1}=\eta_{2}=\eta .
$$

Thus the equations become

$$
\left\{\begin{array}{l}
\frac{d \Sigma_{10}}{d T}=-\Gamma_{10} \\
\left(\Sigma_{10}-\eta \Gamma_{10}\right)\left[1-\sigma\left(\Gamma_{10}+\Gamma_{20}\right)\right]-\kappa_{M} \Gamma_{10}=0 \\
\frac{d \Sigma_{20}}{d T}=-\frac{k_{2}}{k_{1}} \Gamma_{20} \\
\left(\Sigma_{20}-\eta \Gamma_{20}\right)\left[1-\sigma\left(\Gamma_{10}+\Gamma_{20}\right)\right]-\kappa_{M} \Gamma_{20}=0
\end{array}\right.
$$

from which

$$
\begin{gathered}
\Gamma_{10}=\frac{\left.\Sigma_{10}\left[\sigma\left(\Sigma_{20}+\Sigma_{10}\right)+1-\sigma\right)\right]}{2 \eta \sigma\left(\Sigma_{20}+\Sigma_{10}\right)}+ \\
-\frac{\Sigma_{10} \sqrt{\left[\sigma\left(\Sigma_{20}+\Sigma_{10}\right)+(1-\sigma)\right]^{2}-4 \eta \sigma\left(\Sigma_{20}+\Sigma_{10}\right)}}{2 \eta \sigma\left(\Sigma_{20}+\Sigma_{10}\right)} ; \\
\Gamma_{20}=\frac{\Sigma_{20}\left[\sigma\left(\Sigma_{20}+\Sigma_{10}\right)+(1-\sigma)\right]}{2 \eta \sigma\left(\Sigma_{20}+\Sigma_{10}\right)}+ \\
-\frac{\Sigma_{20} \sqrt{\left[\sigma\left(\Sigma_{20}+\Sigma_{10}\right)+(1-\sigma)\right]^{2}-4 \eta \sigma\left(\Sigma_{20}+\Sigma_{10}\right)}}{2 \eta \sigma\left(\Sigma_{20}+\Sigma_{10}\right)} .
\end{gathered}
$$

We find

$$
\begin{array}{r}
\Gamma_{10}=\frac{\Sigma_{10}}{\Sigma_{20}} \frac{\eta_{2}}{\eta_{1}} \Gamma_{20} \quad \Longrightarrow \quad \frac{\Gamma_{10}}{\Gamma_{20}}=\frac{\Sigma_{10}}{\Sigma_{20}} ; \\
\Longrightarrow \quad \frac{d \Sigma_{10}}{d \Sigma_{20}}=\frac{\Gamma_{10}}{\Gamma_{20}} \frac{k_{1} \eta_{1}}{k_{2} \eta_{2}}=\frac{k_{1}}{k_{2}} \frac{\Sigma_{10}}{\Sigma_{20}} .
\end{array}
$$

Since $\lim _{\tau \rightarrow+\infty} s_{10}(\tau)=\lim _{\tau \rightarrow+\infty} s_{20}(\tau)=1$, then, from the matching conditions [2,6-8,32], we have $\Sigma_{10}(0)=\Sigma_{20}(0)=1$. Thus we have

$$
\Sigma_{10}=\left(\Sigma_{20}\right)^{\frac{k_{1}}{k_{2}}}, \quad \Sigma_{20}=\left(\Sigma_{10}\right)^{\frac{k_{2}}{k_{1}}}
$$


From (51), (52) it automatically follows that

$$
\Gamma_{10}(0)=\Gamma_{20}(0)=\frac{(1+\sigma)-\sqrt{\Delta}}{4 \eta \sigma}=\lim _{\tau \rightarrow+\infty} c_{10}(\tau)=\lim _{\tau \rightarrow+\infty} c_{20}(\tau)=\frac{u_{-}}{2} .
$$

Finally, we have the uniform expansions at 0 -th order:

$$
\left\{\begin{aligned}
s_{10}^{u n}(\tau) & =s_{10}(\tau)+\Sigma_{10}(\epsilon \tau)-1=\Sigma_{10}(\epsilon \tau) \\
s_{20}^{u n}(\tau) & =\Sigma_{20}(\epsilon \tau)=\left(\Sigma_{10}(\epsilon \tau)\right)^{k_{2} / k_{1}} \\
c_{i 0}^{u n}(\tau) & =c_{i 0}(\tau)+\Gamma_{i 0}(\epsilon \tau)-\frac{u_{-}}{2}= \\
& =\frac{1}{\eta \sigma}\left[\frac{e^{\sqrt{\Delta} \tau}-1}{u_{+} e^{\sqrt{\Delta} \tau}-u_{-}}\right]+\Gamma_{i 0}(\epsilon \tau)-\frac{u_{-}}{2}
\end{aligned}\right.
$$

Let us observe that the presence of different values of $k_{1}$ and $k_{2}$ is "felt" by the solutions only in the outer contributions, thanks to formula (55).

At 1-st order we have

$$
\begin{cases}\frac{d \Sigma_{11}}{d T} & =-\Gamma_{11} \\ \frac{d \Gamma_{10}}{d T} & =-\sigma\left(\Sigma_{10}-\eta \Gamma_{10}\right)\left(\Gamma_{11}+\Gamma_{21}\right)+\left(\Sigma_{11}-\eta \Sigma_{11}\right)\left[1-\sigma\left(\Gamma_{10}+\Gamma_{20}\right)\right]-\kappa_{M} \Gamma_{11} \\ \frac{d \Sigma_{21}}{d T} & =-\frac{k_{2}}{k_{1}} \Gamma_{21} \\ \frac{d \Gamma_{20}}{d T} & =-\sigma\left(\Sigma_{20}-\eta \Gamma_{20}\right)\left(\Gamma_{11}+\Gamma_{21}\right)+\left(\Sigma_{21}-\eta \Sigma_{21}\right)\left[1-\sigma\left(\Gamma_{10}+\Gamma_{20}\right)\right]-\kappa_{M} \Gamma_{21}\end{cases}
$$

with the appropriate initial conditions, respectively, to be determined by means of appropriate matching conditions.

From (51), (52) we see that

$$
\left\{\begin{aligned}
\Gamma_{10}+\Gamma_{20} & =\frac{1}{2 \eta \sigma}\left[\sigma\left(\Sigma_{10}+\Sigma_{20}\right)+1-\sigma-\sqrt{\left[\sigma\left(\Sigma_{10}+\Sigma_{20}\right)+1-\sigma\right]^{2}-4 \eta \sigma\left(\Sigma_{10}+\Sigma_{20}\right)}\right] \\
\Gamma_{10}-\Gamma_{20} & =\frac{\Sigma_{10}-\Sigma_{20}}{2 \eta \sigma\left(\Sigma_{10}+\Sigma_{20}\right)} \cdot \\
& \cdot\left[\sigma\left(\Sigma_{10}+\Sigma_{20}\right)+1-\sigma-\sqrt{\left[\sigma\left(\Sigma_{10}+\Sigma_{20}\right)+1-\sigma\right]^{2}-4 \eta \sigma\left(\Sigma_{10}+\Sigma_{20}\right)}\right] .
\end{aligned}\right.
$$

Let us set

$$
\begin{array}{llll}
\Sigma_{10}+\Sigma_{20}=X, & \Sigma_{10}-\Sigma_{20}=Y, & \Gamma_{10}+\Gamma_{20}=U, & \Gamma_{10}-\Gamma_{20}=V ; \\
\Sigma_{11}+\Sigma_{21}=W, & \Sigma_{11}-\Sigma_{21}=Z, & \Gamma_{11}+\Gamma_{21}=P, & \Gamma_{11}-\Gamma_{21}=Q .
\end{array}
$$

From (36) and the matching conditions, we have

$$
\Sigma_{11}(0)=s_{11}^{a s}(0)=\frac{-1}{2 \eta \sigma} \ln \left(\frac{\sqrt{\Delta} u_{-}}{2}\right) ; \quad \Sigma_{21}(0)=s_{21}^{a s}(0)=\frac{k_{2}}{k_{1}}\left[\frac{-1}{2 \eta \sigma} \ln \left(\frac{\sqrt{\Delta} u_{-}}{2}\right)\right]
$$

and

$$
\begin{gathered}
W(0)=\left(\Sigma_{11}+\Sigma_{21}\right)(0)=\frac{-1}{2 \eta \sigma}\left(1+\frac{k_{2}}{k_{1}}\right) \ln \left(\frac{\sqrt{\Delta} u_{-}}{2}\right) \\
Z(0)=\left(\Sigma_{11}-\Sigma_{21}\right)(0)=\frac{-1}{2 \eta \sigma}\left(1-\frac{k_{2}}{k_{1}}\right) \ln \left(\frac{\sqrt{\Delta} u_{-}}{2}\right) \\
X(0)=2 \quad ; \quad Y(0)=0 \quad ; \quad \frac{d X}{d T}(0)=-\left(1+\frac{k_{2}}{k_{1}}\right) \frac{u_{-}}{2} \\
\frac{d Y}{d T}(0)=-\left(1-\frac{k_{2}}{k_{1}}\right) \frac{u_{-}}{2} \quad ; \quad U(0)=u_{-} \quad ; \quad V(0)=0 .
\end{gathered}
$$


Moreover, we rewrite (59) in the form

$$
U(T)=\frac{1}{2 \eta \sigma}\left[\sigma X(T)+1-\sigma-\sqrt{[\sigma X(T)+1-\sigma]^{2}-4 \eta \sigma X(T)}\right]
$$

and

$$
V(T)=\frac{Y(T)\left\{\sigma X(T)+1-\sigma-\sqrt{[\sigma X(T)+1-\sigma]^{2}-4 \eta \sigma X(T)}\right\}}{2 \eta \sigma X(T)} .
$$

We have now to obtain $P$ and $Q$. Since, from (58)

$$
\begin{gathered}
\frac{d U}{d T}-(1-\sigma U) W=\left[-\sigma(X-\eta U)-(1-\sigma U) \eta-\kappa_{M}\right] P \\
\frac{d V}{d T}+\sigma P(Y-\eta V)-(1-\sigma U) Z=\left[-(1-\sigma U) \eta-\kappa_{M}\right] Q,
\end{gathered}
$$

we find

$$
\begin{gathered}
P=\frac{(1-\sigma U) W-\frac{d U}{d T}}{\sigma(X-\eta U)+(1-\sigma U) \eta+\kappa_{M}} \\
Q=\frac{\frac{d V}{d T}+\sigma P(Y-\eta V)-(1-\sigma U) Z}{-(1-\sigma U) \eta-\kappa_{M}} .
\end{gathered}
$$

Knowing $U(0), V(0), \frac{d U}{d T}(0), \frac{d V}{d T}(0), X(0), Y(0), W(0), Z(0)$ we can compute $P(0)$ and $Q(0)$. Since, differentiating (64) and (65),

$$
\left\{\begin{aligned}
\frac{d U}{d T}(0) & =-\left(1+\frac{k_{2}}{k_{1}}\right) \frac{u_{-}}{4 \eta}\left[1-\frac{1}{\sqrt{\Delta}}(\sigma+1-2 \eta)\right]= \\
& =-\left(1+\frac{k_{2}}{k_{1}}\right) \frac{u_{-}}{2 \sqrt{\Delta}}\left(1-\sigma u_{-}\right) \\
\frac{d V}{d T}(0) & =\frac{-u_{-}^{2}}{4}\left(1-\frac{k_{2}}{k_{1}}\right)
\end{aligned}\right.
$$

we have

$$
\begin{gathered}
Q(0)=\frac{\left(1-\frac{k_{2}}{k_{1}}\right)}{2\left(\sigma-1+\sigma \eta u_{-}\right)}\left[-\frac{u_{-}^{2}}{2}+\frac{\left(1-\sigma u_{-}\right)}{\eta \sigma} \ln \left(\frac{\sqrt{\Delta} u_{-}}{2}\right)\right] \\
P(0)=-\frac{1}{2 \sqrt{\Delta}}\left(1+\frac{k_{2}}{k_{1}}\right)\left(1-\sigma u_{-}\right)\left[\frac{1}{\eta \sigma} \ln \left(\frac{\sqrt{\Delta} u_{-}}{2}\right)-\frac{u_{-}}{\sqrt{\Delta}}\right]=p^{a s}(0)
\end{gathered}
$$

from which we can obtain back

$$
\Gamma_{11}(0)=\frac{P(0)+Q(0)}{2} \quad ; \quad \Gamma_{21}(0)=\frac{P(0)-Q(0)}{2} .
$$

Observing that, by (32), the following relation

$$
\frac{u_{-}^{2}}{2}=\frac{u_{-}\left(1-\sigma u_{-}\right)}{1-\sigma-\eta \sigma u_{-}}
$$


is identically satisfied by $u_{-}$, we obtain $Q(0)=q^{a s}(0)$, as expected from the matching conditions.

We can finally come back to the original variables, setting

$$
\begin{array}{ccc}
c_{11}(\tau)=\frac{p(\tau)+q(\tau)}{2} \quad ; \quad c_{21}(\tau)=\frac{p(\tau)-q(\tau)}{2} \\
\Gamma_{11}(T)=\frac{P(T)+q(T)}{2} \quad ; \quad \Gamma_{21}(T)=\frac{P(T)-q(T)}{2} .
\end{array}
$$

and obtain the first order correction to the uniform expansions:

$$
\left\{\begin{array}{l}
s_{11}^{u n}=s_{11}(\tau)+\Sigma_{11}(\epsilon \tau)-s_{11}^{a s}(\tau) \\
s_{21}^{u n}=s_{21}(\tau)+\Sigma_{21}(\epsilon \tau)-s_{21}^{a s}(\tau) \\
c_{11}^{u n}=c_{11}(\tau)+\Gamma_{11}(\epsilon \tau)-c_{11}^{a s}(\tau) \\
c_{21}^{u n}=c_{21}(\tau)+\Gamma_{21}(\epsilon \tau)-c_{21}^{a s}(\tau) .
\end{array}\right.
$$
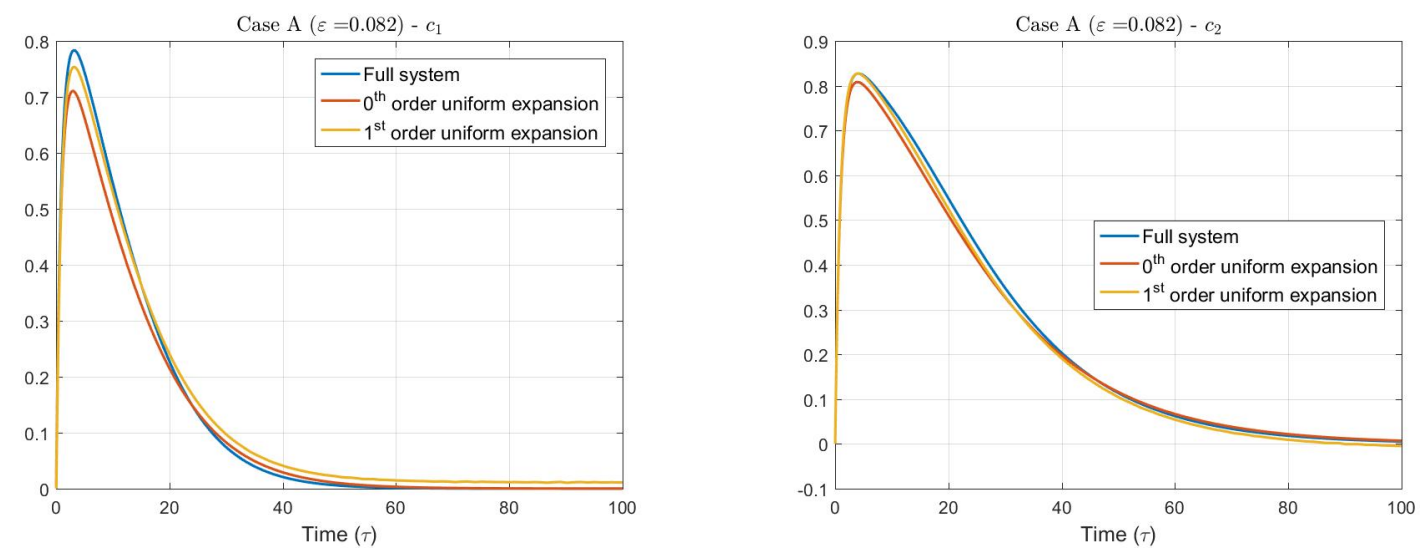

Figure 1. Fully competitive inhibition. Case A. $\epsilon=0.082$ - first order. Comparison between the solutions $c_{1}$ and $c_{2}$ and their uniform 1-st order asymptotic expansions beyond the tQSSA. Though the 0-th order approximation is already satisfactory, in the plots we can anyway clearly observe the effects of the 1-st order correction. Parameters: $a_{1}=k_{1}=1, d_{1}=0.5, K_{1}=$ $1, K_{1 M}=1.5, a_{2}=k_{2}=1, d_{2}=2, K_{2}=1, K_{2 M}=3, E_{0}=1, S_{i 0}=1, \epsilon \cong 0.082, \epsilon_{2}=0.04, \epsilon_{S S}=0.4, \epsilon_{H T A}=1$.
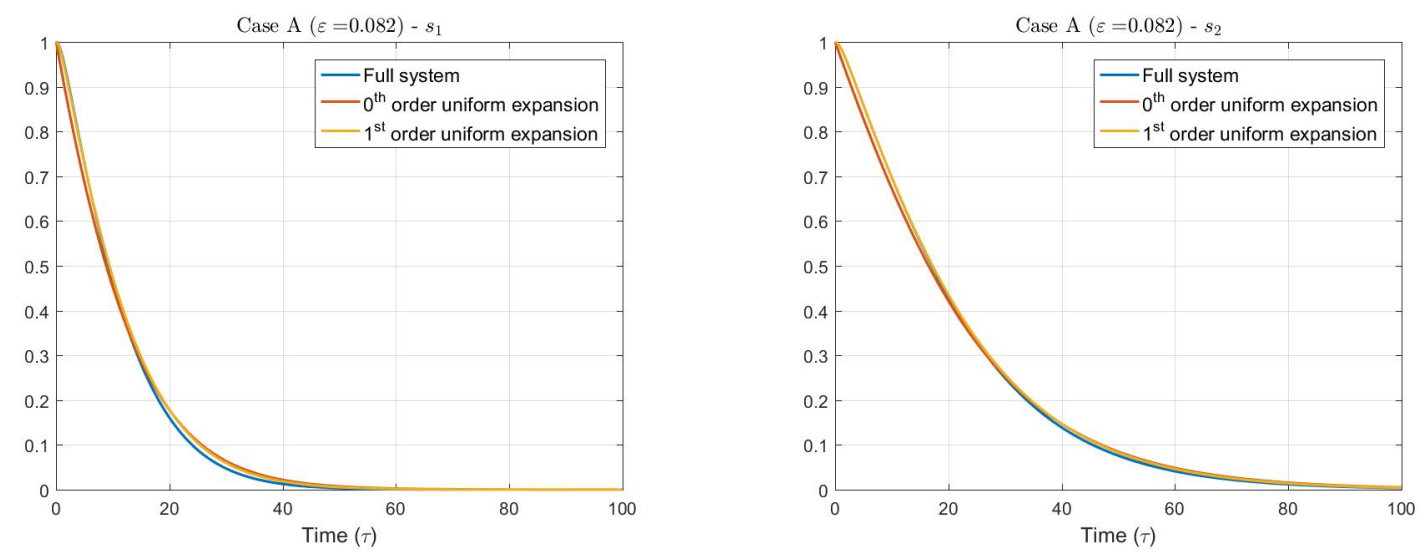

Figure 2. Fully competitive inhibition. Case A. $\epsilon=0.082$ - first order. Comparison between the solutions $s_{1}$ and $s_{2}$ and their uniform 1-st order asymptotic expansions beyond the tQSSA. The 0-th order approximation is already satisfactory. Parameters: $a_{1}=k_{1}=1, d_{1}=0.5, K_{1}=1, K_{1 M}=1.5, a_{2}=k_{2}=1, d_{2}=2, K_{2}=1, K_{2 M}=3, E_{0}=1, S_{i 0}=1, \epsilon \cong$ $0.082, \epsilon_{2}=0.04, \epsilon_{S S}=0.4, \epsilon_{H T A}=1$.

In figures (1)-(2) and (3)-(4) we show the time behaviour of $c_{1}, c_{2}, s_{1}$ and $s_{2}$ in the cases $\epsilon \cong 0.082$ and $\epsilon \cong 0.25$, respectively. 

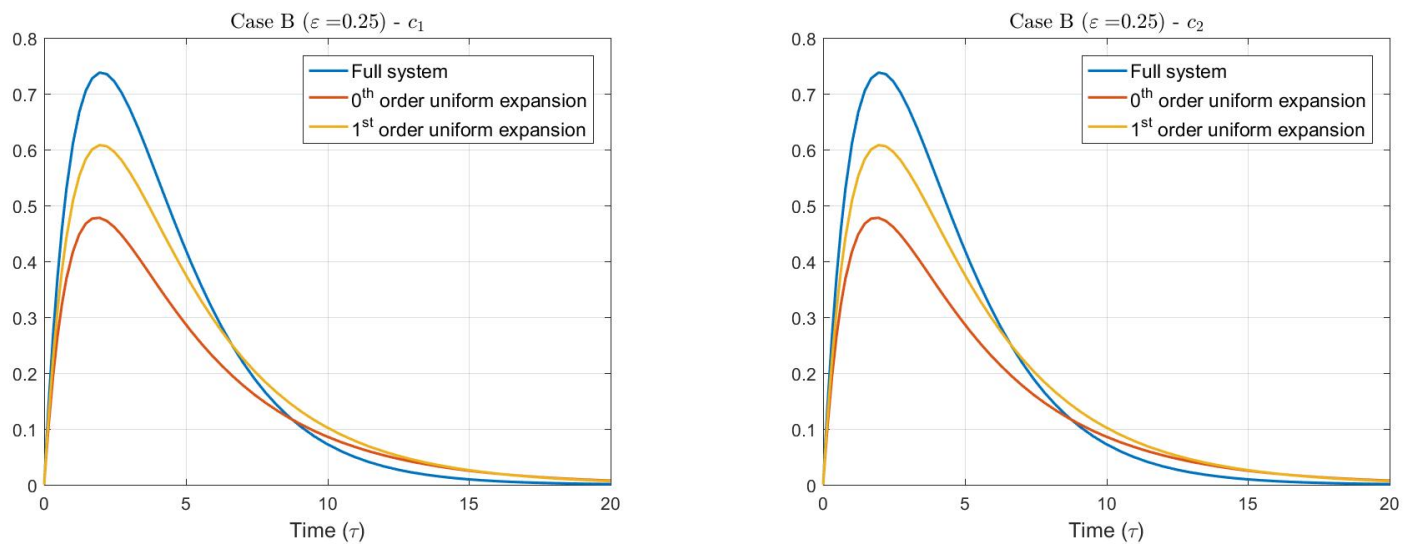

Figure 3. Fully competitive inhibition. Case B. $\epsilon=0.25$ - first order. Comparison between the solutions $c_{1}$ and $c_{2}$ and their uniform 1-st order asymptotic expansions beyond the tQSSA. Since, in this case, $\epsilon=0.25$, in the plots we can clearly observe that the 1-st order corrections are not sufficient to guarantee a satisfactory approximation of the numerical solutions, even if they clearly improve the approximations. Parameters: $a_{i}=k_{i}=1, d_{i}=0.01, K_{i}=1, K_{i M}=1.01, E_{0}=1, S_{i 0}=$ $0.01, \epsilon \cong 0.25, \epsilon_{S S} \cong 1, \epsilon_{H T A}=100$.
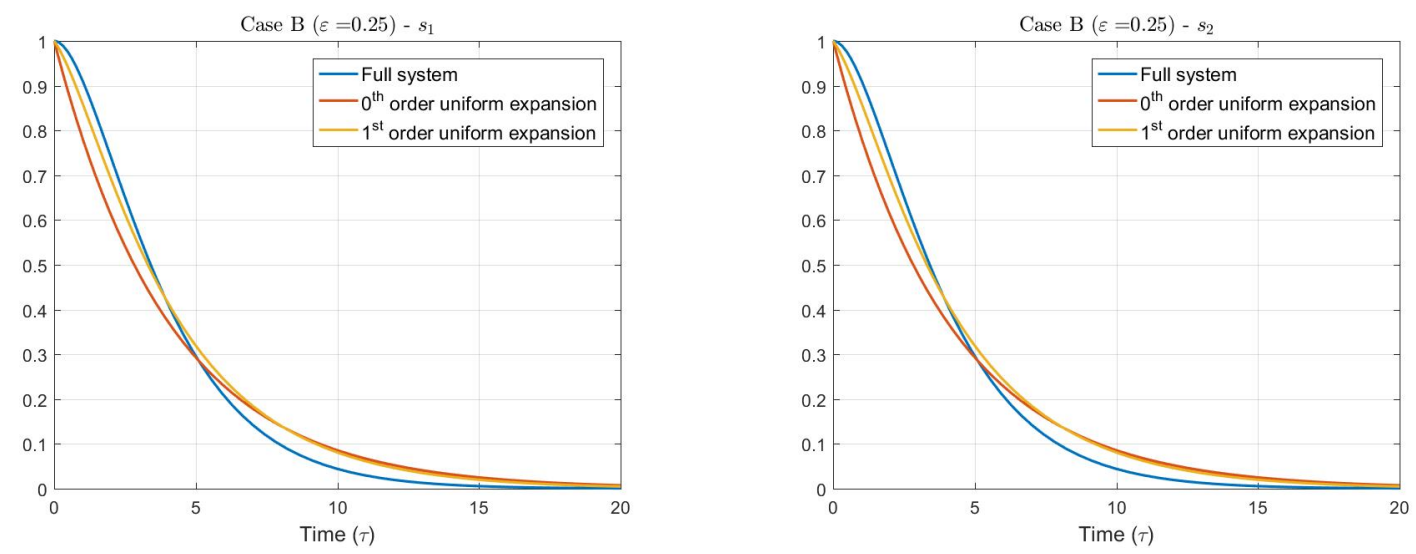

Figure 4. Fully competitive inhibition. Case B. $\epsilon=0.25$ - first order. Comparison between the solutions $s_{1}$ and $s_{2}$ and their uniform 1-st order asymptotic expansions beyond the tQSSA. Though, in this case, $\epsilon=0.25$, in the plots we can appreciate the fact that the 1-st order corrections give a satisfactory approximation of the numerical solutions and clearly improve the approximations. Parameters: $a_{i}=k_{i}=1, d_{i}=0.01, K_{i}=1, K_{i M}=1.01, E_{0}=1, S_{i 0}=0.01, \epsilon \cong 0.25, \epsilon_{S S} \cong 1, \epsilon_{H T A}=$ 100.

The values of parameters and initial conditions are reported in the figure captions.

In the first case the approximation is absolutely satisfactory, mainly for $s_{1}$ and $s_{2}$, while for $c_{1}$ and $c_{2}$, as expected, the 0 -th order approximation can be not completely satisfactory in the matching zone. Nevertheless, we can appreciate the improvement of the approximation from 0-th to 1-st order and the fact that the matching time is obtained with great precision.

In the second case we have stressed the initial conditions and the kinetic parameters in order to obtain $\epsilon \cong 0.2475$, which is very close to the upper bound $\frac{1}{4}$.

In this case we cannot expect a completely efficient approximation. Nevertheless, we can appreciate the improvement of our approximation from the 0-th order to the 1-st order and the detection of the matching time with great precision.

Let us underline that, in the case of the single enzymatic reaction, the sQSSA was obtained in [6] and in [7] as the leading order term of an asymptotic expansion with respect to the parameters $\epsilon_{H T A}=\frac{E_{T}}{S_{T}}$ and $\epsilon_{S S}=\frac{E_{T}}{S_{T}+K_{M}}$, respectively. In the first case explored in this paper $(\epsilon \cong 0.082)$, we would have $\epsilon_{S S}=0.4$ and $\epsilon_{H T A}=1$, which implies that any sQSSA would be unsatisfactory. In the second case 
$(\epsilon \cong 0.25)$, we would have $\epsilon_{S S} \cong 1$ and $\epsilon_{H T A} \cong 100$, implying that any sQSSA would dramatically fail.

\section{Conclusion and perspectives}

In this paper we studied the asymptotic properties of the fully competitive inhibition mechanism. Our studies have been carried out in the tQSSA framework, which proves to be always roughly valid for a broad range of parameter values covering both high and low enzyme concentrations.

Since, as already observed in other papers (see, for example, $[2,6-8]$ ), any QSSA can be interpreted as the leading order term of an asymptotic expansion of the solutions of the ODEs governing the process with respect to an appropriate parameter, the main goal of this paper was to approximate the solutions of the system by asymptotic expansions in terms of a suitable perturbation parameter.

Thus, applying Palsson's technique and Tikhonov's theorem, in a study case we determined the asymptotic expansions up to the 1-st order in $\epsilon$ for the inner and the outer solutions and the corresponding uniform expansions.

We have also given numerical results for different values of the perturbative parameter $\epsilon$ used in the uniform approximations. Numerical results show the influence of the initial concentration of dynamical molecular species and the kinetic constants and guarantee that the predictions made by our analysis are sufficiently accurate.

Our choice of the total approximation has been reinforced by its recent application to complex mechanisms, like the completely reversible enzyme kinetics [34], the antagonist toggle switch [35], the completely competitive inhibition $[26,27,29,36]$, the double phosphorylation [29], the Goldbeter-Koshland switch, which models the single phosphorylation - dephosphorylation cycle [33,37-39], the double phosphorylation - dephosphorylation cycle and the ubiquitous MAPK cascade, which is one of the most important mechanisms present in the great majority of the reaction networks in eukaryotic cells [30,40-44].

In all the cases studied, the tQSSA has shown to be much more efficient than the SQSSA, as the sQSSA predicts phenomena which do not appear when the mechanisms are studied by means of the full system of equations describing the systems or by means of the tQSSA. Representing in any case an approximation, also tQSSA can fail when the system undergoes oscillations, because in this case we cannot expect that the system can reach any equilibrium or quasi-equilibrium state [30].

Palsson's theory seems very promising in order to handle more complex mechanisms by means of Tikhonov theory and Center Manifold Theory. In two works in preparation, we apply these techniques to the double phosphorylation mechanism [45] and the Goldbeter-Koshland switch, or single phosphorylation-dephosphorylation cycle [46].

Let us underline once again the relevant role played by the mechanisms we have focused on in this paper and in $[45,46]$ and the importance of the approximations we adopted to gain qualitative characteristics and to allow biologists to quantify real magnitudes.

Of course several problems are still open. In particular:

In order to determine the asymptotic expansions, we needed in some passages the numerical integration of the equations. However, it would be greatly helpful to find the explicit formulas of all the uniform expansions up to the first order (as done in the sQSSA framework $[27,28]$ ), as they provide such a reliable approximation of the full system. For example, one could make use of the coordinate transformations introduced in [47], in order to yield explicit formulas in terms of generalized Lambert functions also for the outer expansions, at least for the 0-th order (which is the most important, from an experimental viewpoint).

One of the most important consequences of these further studies is the fact that the explicit expressions of these uniform approximations, which can be considered valid at every time, in particular the 0 -th order approximations, can be very useful for fitting the experimental data in any phase (fast transient and quasi-steady state) of the time evolution of the different reactant concentrations in the above described reactions and to determine the values of the parameters characterizing them and that are, in general, not easy to be determined experimentally. 
Let us observe that, for in vitro experimental purposes, it would be sufficient to take values of $E_{T}$ and $S_{T}$ such that the corresponding values of $\epsilon$ are sufficiently small to guarantee a good approximation of the time course of the concentrations. Let us remark once again that our choice of the perturbation parameter is such that $\epsilon$ can be very small for very large parameter ranges.

In fact, if we go back to the figures shown in this chapter, it is astonishing to notice the validity of uniform approximations up to the first order, so that uniform approximations arise as a promising and efficient tool to study even complex mechanisms, besides the individual Michaelis-Menten reaction.

Moreover also approximate formulas are particularly relevant: another important consequence of our results concerns the possibility to describe even complex reaction networks by means of modules representing simpler mechanisms (phosphorylation-dephosphorylation cycle, double phosphorylation reaction, inhibition, etc.) interconnected by inflows and outflows. Explicit, though approximate, formulas can highly help to capture qualitative characteristics of the networks that couldn't be observed by the numerical integration of the equations.

The eventual goal of our and similar works is to give efficient tools to understand the cell behavior and, more importantly, the cell malfunctioning. In fact, the theoretical study of protein networks and the related numerical results can help to identify the "sensitive" nodes (i.e., interactions) the global behavior mainly depends on, and the dose and chemical structure of potential drugs acting into the cell. In this sense, a reliable mathematical modeling can be a valid tool for pharmacological pre-clinical research.

So far we have considered studies on the molecular level. The examples about inhibition and drugs, given in the Introduction, underline that the understanding of the interaction between molecules and pathways is the basis to understand complex biological systems such as cells, tissues, organs, or even the human body.

Thereby our and similar studies can allow a transition from microscopic scale to macroscopic scale, as their applicability has an effective relevance in the understanding of the workings of the human body under normal conditions and in various disease states ( [48]).

\section{Acknowledgements}

The first author is member of the Gruppo Nazionale per la Fisica Matematica (GNFM) of the Istituto Nazionale di Alta Matematica (INdAM).

\section{References}

1. J. Berg, J. Tymoczko, and L. Stryer, Biochemistry. W. H. Freeman New York, 2002.

2. J. Murray, Mathematical Biology: An introduction. Springer-Verlag New York, 2002.

3. J. Borghans, R. de Boer, and L. Segel, Extending the quasi-steady state approximation by changing variables, Bull.Math.Biol., vol. 58, pp. 43-63, 1996.

4. A. M. Bersani, E. Bersani, G. Dell'Acqua, and M. G. Pedersen, New trends and perspectives in nonlinear intracellular dynamics: one century from michaelis-menten paper, Continuum Mechanics and Thermodynamics, vol. 27, no. 4, pp. 659-684, 2015.

5. A. Cornish-Bowden, One hundred years of michaelis-menten kinetics, Perspectives in Science, vol. 4, pp. $3-9,2015$.

6. F. G. Heineken, H. M. Tsuchiya, and R. Aris, On the mathematical status of the pseudo-steady state hypothesis of biochemical kinetics, Math. Biosc., vol. 1, pp. 95-11, 1967.

7. L. A. Segel and M. Slemrod, The quasi steady-state assumption: a case study in pertubation., Siam Rev., vol. 31, pp. 446-477, 1989.

8. G. Dell'Acqua and A. M. Bersani, A perturbation solution of michaelis-menten kinetics in a "total" framework, Journal of Mathematical Chemistry, vol. 50, no. 5, pp. 1136-1148, 2012.

9. J. Carr, Applications of Center Manifold Theory. Springer-Verlag New York, Heidelberg, Berlin, 1981.

10. S. Wiggins, Normally Hyperbolic Invariant Manifolds in Dynamical Systems, vol. 105. Springer-Verlag New York, 1994. 
11. S. Wiggins, Introduction to applied nonlinear dynamical systems and chaos, vol. 2. Springer-Verlag New York, 2003.

12. F. C. Hoppensteadt, Singular perturbations on the infinite interval, Transactions of the American Mathematical Society, vol. 123, no. 2, pp. 521-535, 1966.

13. A. Tikhonov, On the dependence of the solutions of differential equations on a small parameter (in russian), Mat. Sb. (N.S.), vol. 22, no. 2, pp. 193 - 204, 1948.

14. A. Tikhonov, On a system of differential equations containing parameters (in russian), Mat. Sb. (N.S.), vol. 27, pp. 147-156, 1950.

15. A. Tikhonov, Systems of differential equations containing small parameters in the derivatives (in russian), Mat. Sb. (N.S.), vol. 31, no. 3, pp. 575-586, 1952.

16. A. B. Vasil'eva, Asymptotic behaviour of solutions to certain problems involving non-linear differential equations containing a small parameter multiplying the highest derivatives (in russian), Russian Mathematical Surveys, vol. 18, no. 3, pp. 13-84, 1963.

17. W. Wasov, Asymptotic Expansions for Ordinary Differential Equations. Wiley-InterScience, 1965.

18. I. Dvořák and J. Šiška, Analysis of metabolic systems with complex slow and fast dynamics, Bulletin of Mathematical Biology, vol. 51, no. 2, pp. 255-274, 1989.

19. A. Kumar and K. Josić, Reduced models of networks of coupled enzymatic reactions, Journal of Theoretical Biology, vol. 278, no. 1, pp. 87 - 106, 2011.

20. N. Fenichel, Geometric singular perturbation theory for ordinary differential equations, Journal of Differential Equations, vol. 31, no. 1, pp. 53-98, 1979.

21. A. J. Roberts, Model emergent dynamics in complex systems. SIAM, 2015.

22. B. O. Palsson and E. N. Lightfoot, Mathematical modelling of dynamics and control in metabolic networks. i. on michaelis-menten kinetics, Journal of Theoretical Biology, vol. 111, no. 2, pp. 273 302, 1984.

23. B. O. Palsson, R. Jamier, and E. N. Lightfoot, Mathematical modelling of dynamics and control in metabolic networks. ii. simple dimeric enzymes, Journal of Theoretical Biology, vol. 111, no. 2, pp. 303 - 321, 1984.

24. B. O. Palsson, H. Palsson, and E. N. Lightfoot, Mathematical modelling of dynamics and control in metabolic networks. iii. linear reaction sequences, Journal of Theoretical Biology, vol. 113, no. 2, pp. $231-259,1985$.

25. B. O. Palsson, On the dynamics of the irreversible michaelis-menten reaction mechanism, Chemical Engineering Science, vol. 42, no. 3, pp. 447 - 458, 1987.

26. A. Bersani, A. Borri, A. Milanesi, and P. Vellucci, Tihonov theory and center manifolds for inhibitory mechanisms in enzyme kinetics, Communications in Applied and Industrial Mathematics, vol. 8, no. 1, pp. 81-102, 2017.

27. S. Schnell and C. Mendoza, Time-dependent closed form solutions for fully competitive enzyme reactions, Bulletin of Mathematical Biology, vol. 62, no. 2, pp. 321-336, 2000.

28. S. Schnell and C. Mendoza, Closed form solution for time-dependent enzyme kinetics, Journal of Theoretical Biology, vol. 187, no. 2, pp. 207 - 212, 1997.

29. M. G. Pedersen, A. M. Bersani, and E. Bersani, The total quasi-steady-state approximation for fully competitive enzyme reactions, Bulletin of Mathematical Biology, vol. 69, no. 1, pp. 433-457, 2006.

30. M. G. Pedersen, A. M. Bersani, and E. Bersani, Quasi steady-state approximations in complex intracellular signal transduction networks - a word of caution, Journal of Mathematical Chemistry, vol. 43, no. 4, pp. 1318-1344, 2008.

31. S. Schnell and P. Maini, Enzyme kinetics far from the standard quasi-steady-state and equilibrium approximations, Mathematical and Computer Modelling, vol. 35, no. 1-2, pp. 137-144, 2002.

32. C.-C. Lin and L. A. Segel, Mathematics applied to deterministic problems in the natural sciences, vol. 1. Siam, 1988.

33. M. G. Pedersen, A. M. Bersani, E. Bersani, and G. Cortese, The total quasi-steady-state approx- 
imation for complex enzyme reactions, Mathematics and Computers in Simulation, vol. 79, no. 4, pp. 1010 - 1019, 2008.

34. A. Tzafriri and E. Edelman, The total quasi-steady-state approximation is valid for reversible enzyme kinetics, Journal of Theoretical Biology, vol. 226, no. 3, pp. 303 - 313, 2004.

35. M. Sabouri-Ghomi, A. Ciliberto, S. Kar, B. Novak, and J. J. Tyson, Antagonism and bistability in protein interaction networks, Journal of Theoretical Biology, vol. 250, no. 1, pp. 209 - 218, 2008.

36. S. Rubinow and J. L. Lebowitz, Time-dependent michaelis-menten kinetics for an enzyme-substrateinhibitor system, Journal of the American Chemical Society, vol. 92, no. 13, pp. 3888-3893, 1970.

37. A. Bersani, E. Bersani, and L. Mastroeni, Deterministic and stochastic models of enzymatic networksapplications to pharmaceutical research, Computers $\&$ Mathematics with Applications, vol. 55, no. 5, pp. 879 - 888, 2008. Modeling and Computational Methods in Genomic Sciences.

38. A. Ciliberto, F. Capuani, and J. J. Tyson, Modeling networks of coupled enzymatic reactions using the total quasi-steady state approximation, PLOS Computational Biology, vol. 3, pp. 1-10, 032007.

39. M. G. Pedersen and A. M. Bersani, Introducing total substrates simplifies theoretical analysis at nonnegligible enzyme concentrations: pseudo first-order kinetics and the loss of zero-order ultrasensitivity, Journal of Mathematical Biology, vol. 60, no. 2, pp. 267-283, 2010.

40. C. Kwang-Hyun, S. Sung-Young, K. Hyun-Woo, O. Wolkenhauer, B. McFerran, and W. Kolch, Mathematical modeling of the influence of rkip on the erk signaling pathway, in Computational Methods in Systems Biology (C. Priami, ed.), pp. 127-141, Berlin, Heidelberg: Springer Berlin Heidelberg, 2003.

41. S. MacNamara and K. Burrage, Krylov and steady-state techniques for the solution of the chemical master equation for the mitogen-activated protein kinase cascade, Numerical Algorithms, vol. 51, no. 3, pp. 281-307, 2009.

42. G. Dell'Acqua and A. M. Bersani, Quasi-steady state approximations and multistability in the double phosphorylation-dephosphorylation cycle, in Biomedical Engineering Systems and Technologies (A. Fred, J. Filipe, and H. Gamboa, eds.), pp. 155-172, Berlin, Heidelberg: Springer Berlin Heidelberg, 2013.

43. G. Dell'Acqua and A. M. Bersani, Bistability and the complex depletion paradox in the double phosphorylation-dephosphorylation cycle., in BIOINFORMATICS, pp. 55-65, 2011.

44. A. M. Bersani, G. Dell'Acqua, and G. Tomassetti, On stationary states in the double phosphorylationdephosphorylation cycle, AIP Conference Proceedings, vol. 1389, no. 1, pp. 1208-1211, 2011.

45. A. Bersani, A. Borri, A. Milanesi, G. Tomassetti, and P. Vellucci, Asymptotic analysis of the double phosphorylation mechanism, in a tqssa framework. in preparation.

46. A. Bersani, A. Borri, A. Milanesi, G. Tomassetti, and P. Vellucci, Uniform asymptotic expansions beyond the tqssa for the goldbeter-koshland switch. in preparation.

47. A. R. Tzafriri and E. R. Edelman, Quasi-steady-state kinetics at enzyme and substrate concentrations in excess of the michaelis-menten constant, Journal of Theoretical Biology, vol. 245, no. 4, pp. 737 $748,2007$.

48. H. P. Fischer, Mathematical modeling of complex biological systems: from parts lists to understanding systems behavior, Alcohol Research \& Health, vol. 31, no. 1, p. 49, 2008. 